\section{Media Innovations: Success and Paradox.}

\section{Editorial Introduction}

\section{Charles M. Ess}

Department of Media and Communication, University of Oslo

This issue of The Journal of Media Innovation brings together four papers, each of which offers very fine-grained empirical insight into innovation processes - and this from four different countries / cultural domains, namely: Flanders (by authors Jonas De Meulenaere, Lizzy Bleumers, and Wendy Van den Broeck), Spain (David FernándezQuijada, Montse Bonet, Roberto Suárez Candel, and Luis Arboledas), Åland, Finland (Carl-Gustav Lindén); and last, but certainly not least, Bergen, Norway (Lars Nyre). The range of media in play is equally diverse: TV broadcasting in Flanders; Public Service Media in Spain; newspapers (both print and online) in Åland; and radio journalism in Bergen. At the same time, the last three papers share a normative interest in journalism and communication as critical to democratic societies, and, more broadly, continue the focus on innovation in journalism as a primary emphasis of The Journal of Media Innovations (volume 1, issue 2).
The papers can then be compared and contrasted with one another as each offers a distinctive insight into innovation processes with these specific media and cultural contexts. First of all, the papers offer a broad contrast: the initial paper (De Meulenaere et al) offers us something of an innovation success story with regard to so-called second screen usages and applications. By contrast, the remaining three papers highlight the limits and tensional paradox of innovation. That is, while each of these helpfully uncovers specific conditions for successful innovation - each further highlights important constraints: and, as we will see, these constraints fit into a larger pattern in innovation literature - namely, how opportunities for innovation made possible in the first instance by technological innovation are hampered, if not closed down, by what we can think of as more organizational and cultural factors within the specific business operations that otherwise seem eager for innovation (Ess 2014, 3). More fundamentally, as Fernandez-Quijada and his co-authors point out, successful innovation is "basically non-formal," i.e., innovation cannot be "translated into specific formal practices" (32). Indeed, the lack of formal strategies and processes for innovation seems to be a necessary requirement for innovation. On the one hand, this should not be a surprise: insofar as innovation can be defined as what overcomes and transforms previous and well-established - i.e., formulaic - practices, the effort to formulate what overcomes formula is not simply paradoxical but ultimately self-contradictory. On the other hand, especially where academic approaches to innovation aim to establish theories that thus demand logical coherence and consistency - insofar as innovation may prove to be (at least somewhat) intrinsically paradoxical, if not self-contradictory, innovation theories will have to adjust accordingly.

We will return to these broad strokes after first reviewing each article in somewhat more detail.

In our first article, "An Audience Perspective on the Second Screen Phenomenon," De Meulenaere, Bleumers, and Van den Broeck examine a little-researched area - namely, what actually motivates people to make use of second screens, 
i.e., tablets or smartphones, in conjunction with their TV viewing? Their approach is notable first of all for its distinctive method - namely, a conjunction of both sampling and ladder interviewing. This methodology then generates a number of significant findings. First of all, not every sort of TV show is suitable to enhancement via second screen applications, and the authors identify the specific features of TV shows that appear to work best for second screen enhancement. Their informants further identify four important reasons or features of using second screens, including acquiring new information, acquiring new skills, and, perhaps most importantly, experiencing something called "found time". As defined in a Google study (2012), the ability to perform multiple tasks simultaneously e.g., both watching a TV show and looking up an unfamiliar reference or point of curiosity - gives people a sense of more efficient or worthwhile use of time. As well - somewhat counter-intuitively - such multitasking, if carried out properly, can lead to a more cognitively relaxed experience of TV watching, which is, of course, a primary goal in consuming TV.

So far, so good. The terrain - both theoretical and practical - becomes a little more difficult, however, as we turn to our second article. In their "From rhetorics to practice: implementation of technological innovation within Spanish public service media," David Fernández-Quijada and co-authors Suárez, Candel, and Arboledas first demonstrate through their literature review that "innovation" is difficult to define. Hence they stipulate their focus on "the process of content production and distribution within public broadcasters and the structural conditions around it" (27). Their contribution is further distinctive as it offers a comparative approach. As they examine innovation in Public Service Media (PSM), they note that PSM play a key role in innovation more broadly - namely, as they are able to "experiment with services that are not economically profitable" - and in this way, can serve as an innovation test-bed that can contribute to other media organizations as well.

As suggested above, their interviews show that "the unanimous opinion is that innovation is basically non-formal. There can be some planning for specific projects but, generally speaking, the importance given to innovation in the remit and public discourse is not translated into specific formal practices" (32). This leads one informant to observe that "innovation needs order, but also some chaos, to succeed" (32). One technique for getting at this balance between order and chaos is to ex- periment with blurring roles - namely, "the traditional boundaries between journalists, designers and technicians" (32). As we have seen previously, such blurring of roles is indeed an effective strategy - but also one that tends to more feasible for younger rather than more experienced journalists (Nygren 2014). The concluding summary of what works (and what doesn't) in attempting to foster innovation in PSM will be especially important for those interested in seeking to foster innovation in economic environments similar to those of the PSM in Spain - namely, one of the most poorly funded PSM in Europe.

Carl-Gustav Lindén's "Media innovation in a Strange Place: Newspaper Differentiation on Åland," is exceptional first of all for its extensive literature review, one that is most helpful for connecting his findings with the larger literature on innovation. Lindén seeks to examine two key claims - one, that competition among newspapers (and other media) is good for democracy as such competition fosters greater diversity of viewpoint and discussion, and two, what he calls "a linear view of competition between media," i.e., one that argues that such competition will also foster both innovation and differentiation. Clearly, the first thesis depends on the second - but Lindén finds that in his 
study of two newspapers in Åland (the smaller, more liberal - and more innovative - Nya Alland, and the larger, more conservative Ålandstidningen), competition leads "to less, not more differentiation, with highly substitutable goods produced for the same group of consumers" (48). To be sure, the context here is complicated by the fact that both newspapers are owned by the same person: part of his business interest in supporting two competing papers is that it helps lower costs for advertising in both venues, which benefits both the two papers and the advertisers in the region.

Moreover, Lindén finds that organisational culture - i.e., as either more or less open to innovation - is the more important factor than available resources and processes in terms of fostering innovation. Less positively, competition has done nothing to slow down the continued decline in newspaper circulation. At the same time, "innovation in a traditional newsroom does not generate new resources quickly" (48). This makes clear that "a linear view of competition between media," i.e., as fostering innovation and differentiation is simply not true in all instances.

Finally, Lars Nyre offers two prototypes of radio journalism - more precisely, what he calls "hyperlocal journalism" - in his "Designing the
Amplifon.” Nyre shares with Lindén a normative interest in innovation in information and communication technologies (ICTs) that foster communication for the public good - an interest also shared, if more implicitly, in Fernandéz_Quijada et al. For his part, Nyre draws on John Dewey's understanding of how communication is critical to "the public" in a democratic sense (cf. Nyre 2014). More specifically, the two prototypes described here - labelled by the author as "Walk-by reportage" and "Sitting in the train-news" are designed to exploit the possibilities of both locative media and "smart" (i.e., noise-cancelling) headphones. Insofar as either of these might be taken up by a Public Service Broadcaster such as the Norwegian Broadcasting Corporation (NRK), they would thereby represent dramatic new extensions of the PSB / PSM remits.

At the same time, however, Nyre recognizes that such designs typically cannot be expected to become realized in these ways for as long as eight to ten years - if ever. This is in part because of the new sorts of demands and transformations in journalistic practices and genres that these prototypes require. In this way, Nyre reinforces what is now a commonplace in this journal, beginning with the Research Brief by Jan Bierhoff and Sander Kruitwagen on "the Reading Radar" (2014). Again, it is precisely as new technologies make innovations in journalism possible that they thereby clash with established practices. Whether these practices are justified by the quality of their results, and/or by the human tendency to remain with what is comfortable and familiar - and certainly with what is demonstrated to be profitable vs. the uncertainties of new practices and products - one or more of these factors often successfully conspire against the successful introduction of the innovation (cf. Ess 2014, 3).

This means that we may never see - or more precisely, hear - Nyre's prototypes become a regular part of our journalistic diet. In my view, this would be a pity, precisely given how these forms of hyperlocal journalism might well contribute to the normative goods of expanding communicative sources and practices in ways that can contribute to "the public" in Dewey's sense.

More broadly, taken together these papers demonstrate not only that innovation in media - specifically journalistic media - remains "poorly defined and not well understood" (Bleyen, et al. 2014 28), beginning within an academic or theoretical perspective. Within praxis as well, attempting to make innovation work often encounters more ob- 
stacles than success, as especially the last three articles detail. Nonetheless, we can adopt Gayatri Chakravorty Spivak on the profound difficulties of capturing culture alive, as "always on the run, always changeful ... There is no reason to throw up one's hands over this" (1999, 356f.). Especially given that media innovations is still very young academically, it is not surprising that canonical definitions and theories have yet to be fully articulated. ${ }^{1}$

At the same time, of course, identifying sources of failure thereby provides critical findings that hold up success stories such as those presented here by De Meulenaere et al in greater relief. And so some measure of progress may be discerned.

1 As a point of comparison: "Internet Studies" emerged out of diverse disciplines in the early 1990s, to begin finding its academic expressions in flagship journals and then organizations such as the Association of Internet Studies (AoIR, 2000) and institutions such as the Oxford Internet Institute (founded 2001). Only within the past few years have there emerged efforts to develop "theories of the Internet" (e.g., Ropolyi 2013, Panayiota 2014). My impression is that innovation studies are roughly where Internet Studies was ca. 2000 - just on the cusp of becoming more institutionalized in what we can hope will be significant and lasting ways.
To conclude the issue, Lars Nyre reviews Adriana de Souza e Silva and Mimi Sheller (Eds), Mobility and Locative Media: Mobile Communication in Hybrid Spaces (Routledge: 2014).As always, a thousand thanks are due to our many reviewers and the Editorial Team that makes the Journal run - namely: Anders Olof Larsson (Editorial Assistant), Anders Fagerjord (Layout Editor), and Arne H. Krumsvik (Book Review Editor).

\section{REFERENCES}

Bierhoff, Jan, and Kruitwagen, Sander. 2014. Stories behind the News; Designing an Advanced App for Journalistic Background Information. Journal of Media Innovations 1 (1): 110-128. DOI: http://dx.doi. org/10.5617/jmi.v1i1.770

Bleyen, Valérie-Anne, Lindmark, Sven, Ranaivoson, Heritiana, and Ballon, Pieter. 2014. A Typology of Media Innovations: Insights from an Exploratory Study. Journal of $\mathrm{Me}$ dia Innovations 1 (1): 28-51. DOI: http:// dx.doi.org/10.5617/j

Ess, Charles. 2014. Editor's Introduction: Innovations in the newsroom - and beyond. The Journal of Media Innovations 1 (2): 1-9. DOI: http://dx.doi.org/10.5617/jmi. v1i2.923

Nygren, Gunnar. 2014. Multiskilling in the newsroom - de-skilling or re-skilling of journalistic work? Journal of Media Innovations 1 (1): 75-96. DOI: http://dx.doi.org/10.5617/ jmi.vii2.876.

Nyre, Lars. 2014. Media design method. Journal of Media Innovations 1 (1): 86-109. http:// dx.doi.org/10.5617/jmi.v1i1.702 
Panayiota, Tsatsou. 2014. Internet Studies: Past, Present, and Future Directions. Farnham, Surrey: Ashgate.

Ropolyi, László. 2013. Philosophy of the Internet: A Discourse on the Nature of the Internet. $<$ http://elte.prompt.hu/sites/default/files/ tananyagok/PhilosophyOfTheInternet/index.html>

Spivak, Gayatri Chakravorty. 1999. A Critique of Postcolonial Reason: Toward a History of the Vanishing Present. Cambridge, Mass.: Harvard University. Press. 